\title{
Circulating miR-200 family as predictive markers during systemic therapy of metastatic breast cancer
}

\author{
Chiara Fischer $^{1} \cdot$ Thomas M. Deutsch $^{1}$ (1) $\cdot$ Manuel Feisst $^{2} \cdot$ Nathalie Rippinger $^{1} \cdot$ Fabian Riedel $^{1}$. \\ Andreas D. Hartkopf ${ }^{3} \cdot$ Sara Y. Brucker ${ }^{3} \cdot$ Christoph Domschke $^{1} \cdot$ Carlo Fremd $^{4} \cdot$ Laura Michel $^{4} \cdot$ Barbara Burwinkel $^{5}$. \\ Andreas Schneeweiss $^{4,5} \cdot$ Andrey Turchinovich $^{5} \cdot$ Markus Wallwiener $^{1}$
}

Received: 28 July 2021 / Accepted: 10 February 2022 / Published online: 2 March 2022

(c) The Author(s) 2022

\begin{abstract}
Purpose Circulating miRNAs can provide valid prognostic and predictive information for breast cancer diagnosis and subsequent management. They may comprise quintessential biomarkers that can be obtained minimally invasively from liquid biopsy in metastatic breast cancer patients. Therefore, they would be clinically crucial for monitoring therapy response, with the goal of detecting early relapse. This study investigated miRNA expression in patients with early and/or late relapse, and the predictive value for assessing overall (OS) and progression-free survival (PFS).

Methods Forty-seven patients with metastatic breast cancer from the University Women's Hospital Heidelberg were enrolled in this study. Expression of miR-200a, miR-200b, miR-200c, miR-141, and miR-429 was analyzed by RT-qPCR before a new line of systemic therapy and after the first cycle of a respective therapy. Tumor response was assessed every 3 months using the RECIST criteria. Statistical analysis focused on the relation of miR-200s expression and early vs. late cancer relapse in relation to systemic treatment. The association of miRNAs with PFS and OS was investigated.

Results Before starting a new line of systemic therapy, miR-429 $(p=0.024)$ expression was significantly higher in patients with early relapse (PFS $\leq 4$ months) than in patients with late relapse (PFS $>4$ months). After one cycle of systemic therapy, $\operatorname{miR}-200 \mathrm{a}(p=0.039)$, miR-200b $(p=0.003)$, miR-141 $(p=0.017)$, and miR-429 $(p=0.010)$ expression was higher in early than in late progressive cancer. In addition, 4 out of $5 \mathrm{miR}-200$ family members (miR-200a, miR-200b, miR-141, and miR429) predicted PFS ( $p=0.048, p=0.008, p=0.026$, and $p=0.016$, respectively). Patients with heightened miRNA levels showed a significant reduction in OS and PFS.

Conclusion Circulating miR-200s were differentially expressed among patients with late and/or early relapse. 4 of 5 members of the miR-200 family predicted significantly early relapse after systemic treatment. Our results encourage the use of circulating miR-200s as valuable prognostic biomarkers during metastatic breast cancer therapy.
\end{abstract}

Keywords Metastatic breast cancer $\cdot$ Liquid biopsy $\cdot$ Circulating microRNAs $\cdot$ miR-200 family $\cdot$ Survival

\section{Introduction}

Breast cancer is the most frequently diagnosed cancer entity worldwide, with about 2.1 million new cases having been

Chiara Fischer, Thomas M. Deutsch, Andrey Turchinovich and Markus Wallwiener contributed equally. predicted for 2018 a quarter (24.2\%) of all cancer cases

Thomas M. Deutsch

thomas.deutsch@med.uni-heidelberg.de

1 Department of Obstetrics and Gynecology, University of Heidelberg, Im Neuenheimer Feld 440, 69120 Heidelberg, Germany

2 Institute of Medical Biometry, University of Heidelberg, Im Neuenheimer Feld 130.3, 69120 Heidelberg, Germany
3 Department of Obstetrics and Gynecology, University of Tübingen, Calwerstraße 7, 72076 Tübingen, Germany

4 National Center for Tumor Diseases, Im Neuenheimer Feld 460, 69120 Heidelberg, Germany

5 Molecular Epidemiology C080, German Cancer Research Center (DKFZ), Im Neuenheimer Feld 581, 69120 Heidelberg, Germany 
among women [1]. In 2018, Germany anticipated 71,900 new cases of breast cancer and a total of 18,136 breast cancer-related deaths [2].

Metastatic breast cancer constitutes a palliative scenario requiring an entirely different treatment approach that meticulously focuses on symptom management and improving patients' quality of life as opposed to measures for prolonging life. Thus, it is of utmost importance to provide treatment according to a patient's stage of disease and the previously specified prognosis, thereby preventing unnecessary suffering due to inappropriate therapeutic measures and/or lack of treatment. Here, biomarkers are required that can be easily obtained and reliably reflect the current tumor burden and prognosis.

Current research emphasizes the role of readily obtainable tumor-derived blood components that have predictive and prognostic value. Such components include circulating microRNA (miRNA), circulating tumor cells, cell-free DNA, circulating tumor DNA, circulating tumor RNA, exosome vesicles, and tumor-educated platelets [3].

miRNAs constitute small noncoding RNA molecules that regulate the expression of multiple genes by initiating translational silencing and/or degrading their respective cognate mRNA targets [4]. The miR-200 family (miR-200a, miR200b, miR-200c, miR-141, and miR-429) is involved in the dynamic and reversible process of epithelial-mesenchymal transition (EMT), by means of enhancing the expression of E-cadherin and maintaining the epithelial cell phenotype [5]. EMT is a key mechanism in breast cancer progression, as it modulates cell plasticity and plays a crucial part in the invasion and dissemination of tumor cells. During early metastatic events, low levels of miR-200s promote EMT. Thereby, they enable cancer cells to acquire a mesenchymal phenotype as well as the ability to migrate and invade surrounding tissues. Conversely, in late metastatic stages, high levels of miR-200s facilitate mesenchymal-epithelial transition (MET) by promoting the expression of epithelial cell phenotypes. That process is prominently involved in promoting successful colonization in previously infiltrated organs $[6,7]$. For example, related research findings reported high miR-200c levels in blood plasma of patients with advanced breast cancer [8]. Furthermore, high levels of circulating miR-200s measured in these patients' blood samples have been found to correlate with decreased overall (OS) and progression-free survival (PFS) [9-11]. Moreover, clustered analyses, including miR-200c levels, lymph node infiltration, tumor grade, and estrogen receptor were able to predict late relapse [12]. Noticeably and enticingly, recently published findings reported that high levels of miR-200a can predict response to chemotherapy [13]. These findings imply that miRNAs may offer an invaluable opportunity to reliably predict patient outcome and/or response to specific treatment protocols that are currently being followed.
The present analysis aims to provide valuable insights related to the clinical applicability of liquid biopsies as a minimally invasive method for identifying high-risk patient populations in metastatic breast cancer. Hence, our aim is to potentially provide a platform for capturing patients in need of tailored therapeutic approaches in line with their respective overall risk for relapse/recurrence of disease parameters, with the overall goal of maintaining a higher quality of life while combating disease.

\section{Patients and methods}

\section{Study design and samples}

We conducted a retrospective, single-center, cohort study at the National Center for Tumor Diseases (NCT), Heidelberg, Germany, together with the German Cancer Research Center (DKFZ), Heidelberg, Germany, and the Department of Obstetrics and Gynecology, University of Heidelberg, Heidelberg, Germany. This study was approved by the Ethics Committee of the Medical Faculty Heidelberg of the Heidelberg University, approval No. S-295/2009. Written informed consent was given by all participants.

In all, 47 metastatic breast cancer patients $\geq 18$ years who were about to begin with a new line of systemic therapy were consecutively enrolled between April 2010 and September 2011. Previous therapy as well as the time of initial diagnosis were disregarded.

At study entry, radiological evaluation was performed and then repeated every 3 months to classify therapy response according to RECIST criteria [14]. Survival (OS, PFS) was measured in terms of time, specifically so in months, from first blood draw after inclusion in the study and up until progression of disease, death, or loss to follow-up. Furthermore, patients were divided into two prognostic groups according to their previously established RECIST status as obtained during initial radiological assessment at the time of treatment initiation. Initially, the study set out to measure therapeutic success 3 month postinitial intervention. However, as obtaining relevant clinical markers, such as blood samples, or initiation of treatment protocol occurred with up to a week's worth of delay, the authors found it sensible to permit assessment after 4 months in order not to unnecessarily reduce sample size and/or facilitate loss of participants. 4 month postinclusion in the study, patients displaying signs of disease progression according to radiological evaluation were grouped as such (PFS $\leq 4$ months, $n=22$ ) as were those with stable disease or partial/complete response (PFS $>4$ months, $n=25$ ). All patients were of female sex and Caucasian. 


\section{miRNA assessment}

Blood samples from patients with metastatic breast cancer were taken prior to initiating a new line of systemic treatment (baseline) and after one cycle of systemic therapy (three cycles of hormone therapy, respectively) to determine miR-200a, miR-200b, miR-200c, miR-141, and miR-429 expression levels.

\section{Isolation of circulating miRNAs}

A total of $7.5 \mathrm{ml}$ of peripheral blood was collected in $9 \mathrm{ml}$ EDTA tubes (Sarstedt S-Monovette ${ }^{\circledR}$, Nürnbrecht, Germany) and processed within $2 \mathrm{~h}$ according to a two-step centrifugation protocol: $1300 \mathrm{~g}$ for $20 \mathrm{~min}$ at $10{ }^{\circ} \mathrm{C}$, followed by $15,500 \mathrm{~g}$ for $10 \mathrm{~min}$ at $10^{\circ} \mathrm{C}$. Afterwards, samples were snap-frozen and stored at $-80{ }^{\circ} \mathrm{C}$. miRNAs were extracted from $400 \mu$ l of plasma using TRI-Reagent LS ${ }^{\circledR}$ (SigmaAldrich, St. Louis, USA) and Qiagen miRNeasy ${ }^{\circledR}$ mini kit (Qiagen, Hilden, Germany), as described by Turchinovich et.al. [15].

All laboratory procedures were performed at room temperature (RT). First, $400 \mu \mathrm{l}$ blood plasma was denatured in $2 \mathrm{ml}$ Eppendorf tubes by adding 1,200 $\mu \mathrm{l}$ TRI-Reagent LS $^{\circledR}$. Then, 1 pg synthetic cel-miR-39 (spiked-in normalization reference) and $1 \mu \mathrm{l}$ glycogen $(20 \mathrm{mg} / \mathrm{ml})$ (Thermo Scientific, Life Technologies, Carlsbad, USA) were added. Samples were vortexed for $15 \mathrm{~s}$ and incubated for $20 \mathrm{~min}$ at RT. After addition of $220 \mu \mathrm{l}$ chloroform the samples were vortexed for $15 \mathrm{~s}$, incubated for $5 \mathrm{~min}$ at RT, and centrifuged at $16,000 \mathrm{~g}$ for $20 \mathrm{~min}$. The $600 \mu \mathrm{l}$ of upper aqueous phase containing dissolved RNA was collected, mixed with $900 \mu \mathrm{l}$ of $100 \%$ ethanol (Roth Chemicals, Karlsruhe, Germany) and incubated for $5 \mathrm{~min}$ at RT. Afterwards, the RNA was purified using miRNeasy ${ }^{\circledR}$ mini spin columns (Qiagen, Hilden, Germany) according to the manufacturer's instructions. Ultimately, the RNA was eluted in $60 \mu \mathrm{l}$ RNAse-free water and stored at $-80^{\circ} \mathrm{C}$ until further processing.

\section{Quantitative real-time PCR}

Current analysis was based on analyzing a panel of $13 \mathrm{miR}$ NAs developed and validated within a previously published study by Madhavan and colleagues in 2012 [9].

The analyzed miRNAs included: miR-16, miR-24, miR29a, miR-138-5p, miR-141, miR-200a, miR-200b, miR200c, miR-210, miR-375, miR-365, miR-429, miR-1260, as well as exogenous synthetic spike-in normalizer cel-miR-39.

In total, $5 \mu$ l of purified total RNA from $60 \mu \mathrm{l}$ eluate was used as an input into a reverse transcription (RT) reaction performed by TaqMan ${ }^{\circledR}$ MicroRNA Reverse Transcription Kit (Applied Biosystems, Carlsbad, USA). Every RT reaction having $15 \mu \mathrm{l}$ volume in total comprised $5 \mu \mathrm{l}$ of purified
miRNA sample, $3 \mu \mathrm{l}$ of stem-loop RT primers mix ( 3 per RT sample), $1.5 \mu \mathrm{l}$ of RT buffer 10X, $0.15 \mu \mathrm{l}$ of dNTPs $100 \mathrm{mM}$, $0.19 \mu \mathrm{l}$ of RT inhibitor, $1 \mu \mathrm{l}$ of MultiScribe Reverse Transcriptase $50 \mathrm{U} / \mu \mathrm{l}$, and $4.19 \mu \mathrm{l}$ RNase-free water. The RT reactions were incubated on 96-well PCR plates for $30 \mathrm{~min}$ at $16^{\circ} \mathrm{C}$, followed by $30 \mathrm{~min}$ at $42{ }^{\circ} \mathrm{C}, 5 \mathrm{~min}$ at $85^{\circ} \mathrm{C}$, and then held at $4{ }^{\circ} \mathrm{C}$. Afterwards, RT products were diluted in RNase-free water and stored at $-20^{\circ} \mathrm{C}$.

Subsequent qPCR reactions (having final volumes of $10 \mu \mathrm{l} \mathrm{each}$ ) included $2 \mu \mathrm{l}$ of diluted RT product, $5 \mu \mathrm{l}$ of TaqMan ${ }^{\circledR}$ Universal PCR Master Mix (Applied Biosystems, Carlsbad, USA), $0.5 \mu \mathrm{l}$ of corresponding miRNA assay primers and $2.5 \mu \mathrm{l}$ RNase-free water. The qPCR reactions were incubated in LightCycler ${ }^{\circledR} 480$ 384-multiwel plates (Roche Diagnostics, Mannheim, Germany) at $95{ }^{\circ} \mathrm{C}$ for $10 \mathrm{~min}$, followed by 50 cycles of $95^{\circ} \mathrm{C}$ for $10 \mathrm{~s}$ and $60^{\circ} \mathrm{C}$ for $1 \mathrm{~min}$. All reactions were run in duplicate. Real-time PCR was performed using LightCycler ${ }^{\circledR} 480$ Real-Time PCR System (Roche Diagnostics, Mannheim, Germany), and crossing points $(\mathrm{Cp})$ were determined by the second derivative max method implemented in LightCycler ${ }^{\circledR} 480$ software. Relative quantities of miRNA were calculated using a modified $\Delta \Delta$ Ct method after normalization to the cel-miR-39 spiked-in control. Specifically, the normalization factor was calculated using the difference between the mean cel-miR-39 Cp values from all samples and the mean cel-miR-39 Cp values from a respective patient sample.

\section{Statistical analysis}

Demographic data and tumor characteristics were presented as mean, median and range for continuous variables, and in absolute and relative frequencies for categorical variables.

Boxplots displayed differences in miRNA expression levels between patient groups with early and late PFS. Student's $t$ test was performed to investigate differences in miRNA expression levels between patients with early and late progression of disease. Furthermore, Student's $t$ test was performed to assess the miRNA expression in groups with the following characteristics: chemotherapy, endocrine therapy, bone, local and visceral metastasis, respectively. Thereby, normal distribution of the miRNAs could be assumed due to the previous normalization of the miRNA values. Univariable logistic regression analysis was performed to investigate miRNA expression patterns during one cycle of systemic therapy as a predictor for early and/or late disease progression. Multivariable logistic regression analysis included the following variables: age at initial diagnosis, age at study enrollment, tumor receptor status (HR+/ HER2-, HER2+, TNBC), chemotherapy, endocrine therapy as well as visceral, local and bone metastasis, respectively. Kaplan-Meier curves were generated alongside univariable Cox-regressions and corresponding hazard ratios with $95 \%$ 
confidence intervals to evaluate patient outcome. To calculate and compare survival probability, the miRNA Cp values were dichotomized into patient groups with lower and higher miRNA expression, defined as one lower quartile and three upper quartiles [9]. The level of significance was set at alpha 5\%. Due to the exploratory character of this study, $p$ values have to be interpreted in a descriptive sense and are not adjusted for multiplicity. No missing values have been imputed. Statistical analysis was performed using $\mathrm{R}$ version 3.5.1 (2018-07-02) [16].

\section{Results}

In all, 47 consecutive patients were enrolled in this study. Patient characteristics and tumor biology at baseline are displayed in Table 1. Of the respective 47 patients, 22 suffered progression of disease within 4 months after one cycle of chemotherapy (three cycles of hormone therapy, respectively), whereas 25 experienced disease progression after more than 4 months. The median time interval between the analysis of miRNAs before starting a new systemic therapy and after one cycle of the respective therapy was 41.5 days (Range: 26-70) in patients with PFS $\leq 4$ months and 40 days (26-149) in patients with PFS $>4$ months, respectively.

Figures 1 and 2 illustrate expression levels of circulating miR-200a, miR-200b, miR-200c, miR-141, and miR-429 in patient groups with either early and/or late disease progression, both before and after one cycle of systemic treatment. At both timepoints, plasma miRNA levels tended to be higher in patients with early disease progression than in patients with late disease progression. Before initiating a new line of systemic therapy, only expression of miR429 was significantly higher in early progressive cancer $(p=0.024)$. After therapeutic intervention, however, miR200a, miR-200b, miR-141, and miR-429 expression levels were significantly higher in patients with early progression $(p=0.039, p=0.003, p=0.017, p=0.010)$. miR-200c was marginally not statistically significant $(p=0.076)$ (Table 2$)$. Thereafter, univariable logistic regression analysis was utilized to analyze the aforementioned circulating miRNAs in terms of their predictive value related to PFS. Circulating miRNA levels after systemic treatment showed that miR200a, miR-200b, miR-141, and miR-429 could predict probability of PFS. Logistic regression analysis of miR-200c and PFS, however, marginally did not reach the level of statistical significance (Table 3).

A potential biasing influence of systemic chemotherapy or endocrine therapy on miRNA expression was investigated by Student's $t$ tests. These showed no systematic differences in miRNA expression between patients with and without chemotherapy. Only the expression levels of miR429 in patients with endocrine therapy differed significantly after systemic therapy $(p=0.02)$. Regarding, the influence of metastatic disease, miRNA expression was different in some miRNAs in patients with local metastasis (baseline: miR-200a, miR-200b, $p \leq 0.05$; after systemic therapy: miR-200a, miR-200c, miR-141, $p \leq 0.02$ ) and bone metastasis (baseline: miR-200a, miR-200b, miR-200c, miR-141, miR-429, $p \leq 0.02$; after systemic therapy: miR-200a, miR200b, miR-200c, miR-141, $p \leq 0.002$ ) at baseline and after systemic therapy (supplementary material, Table 1).

In a multivariable Cox-regression model for further assessment of confounding variables, miR-200a $(p=0.037)$, miR-200b $(p=0.048)$, and miR-141 $(p=0.041)$ continued to predict early progression of disease at a statistically significant level (supplementary material, Table 2). Neither miR-200c $(p=0.132)$, miR-429 $(p=0.073)$, nor any of the potentially confounding variables accounted for in the analysis reached statistical significance, including the metastatic location. Regarding patient outcome, average PFS in patients with PFS $\leq 4$ months was 2.6 (Range: $1-4$ ) months, compared to $17.6(5-48)$ months in patients with PFS $>4$ months. Mean OS was 15.659 (3-54) months in patients with PFS $\leq 4$ months vs. 38.2 (10-84) months with PFS $>4$ months, respectively (Table 1 ). The comparison of survival probabilities of patients with high vs. low miRNA levels according to Kaplan-Meier curves indicated significantly lower OS and PFS among patients with heightened expression levels of miR-200a, miR-200b, miR-200c, miR141, and miR-429, respectively (Figs. 3 and 4). Cox-regression and hazard ratios validated these results, comparing OS and PFS distribution among these prognostic groups (Table 4). Moreover, miRNAs were not only significantly related to PFS and OS as dichotomized but also as continuous variable, further strengthening their value as prognostic marker (supplementary material, Table 3).

\section{Discussion}

This study aimed to investigate circulating EMT-specific miRNA levels in the blood of metastatic breast cancer patients during and after one cycle of systemic therapy to determine potential liquid biomarkers for monitoring both cancer progression and treatment efficacy.

Heightened expression of miR-200a, miR-200b, miR200c, miR-141, and miR-429 is known to be associated with reduced OS and PFS probability $[9,11,17]$.

The present study was able to replicate published findings by showing a significant relation between unfavorable outcome and heightened miR-200a, miR-200b, miR-200c, miR-141, and miR-429 expression. Elevated plasma levels of the aforementioned miRNAs were significantly related to worse OS and PFS after one cycle of systemic therapy. This finding is especially compelling as prognostic evaluations 
Table 1 Characteristics of patients with early (PFS $\leq 4$ months) and late (PFS $>4$ months) relapse

\begin{tabular}{|c|c|c|c|c|}
\hline \multirow[t]{2}{*}{ Characteristic } & \multicolumn{2}{|c|}{ Early (PFS $\leq 4$ months) } & \multicolumn{2}{|c|}{ Late (PFS > 4 months) } \\
\hline & Number & Percentage & Number & Percentage \\
\hline Number of patients & 22 & 46.8 & 25 & 53.2 \\
\hline \multicolumn{5}{|l|}{ Age at initial diagnosis (years) } \\
\hline Mean & 47 & & 52 & \\
\hline Range & $33-72$ & & $34-77$ & \\
\hline \multicolumn{5}{|l|}{ Age at baseline (years) } \\
\hline Mean & 54 & & 60 & \\
\hline Range & $35-89$ & & $40-47$ & \\
\hline NST & 40 & 45.5 & 11 & 44.0 \\
\hline ILC & 4 & 18.2 & 4 & 16.0 \\
\hline Other & 0 & 0 & 1 & 4.0 \\
\hline Unknown & 8 & 36.4 & 9 & 36.0 \\
\hline HR positive / HER2 negative & 9 & 40.9 & 12 & 48.0 \\
\hline HER2 positive & 1 & 4.5 & 4 & 16.0 \\
\hline TNBC & 4 & 18.2 & 1 & 4.0 \\
\hline \multicolumn{5}{|c|}{ Distant metastasis at initial diagnosis } \\
\hline No & 14 & 63.4 & 15 & 60.0 \\
\hline Yes & 5 & 22.7 & 5 & 20.0 \\
\hline Unknown & 3 & 13.6 & 5 & 20.0 \\
\hline \multicolumn{5}{|l|}{ Overall survival } \\
\hline Median & 15.6 & & 38.2 & \\
\hline Range & $3-54$ & & $10-84$ & \\
\hline \multicolumn{5}{|l|}{ Progression-free survival } \\
\hline Median & 2.6 & & 17.6 & \\
\hline Range & $1-4$ & & $5-48$ & \\
\hline \multicolumn{5}{|l|}{ Visceral metastasis } \\
\hline Yes & 14 & 63.6 & 11 & 44.0 \\
\hline No & 8 & 36.4 & 14 & 56.0 \\
\hline \multicolumn{5}{|l|}{ Local metastasis } \\
\hline Yes & 9 & 40.1 & 14 & 56.0 \\
\hline No & 13 & 59.1 & 11 & 44.0 \\
\hline \multicolumn{5}{|l|}{ Bone metastasis } \\
\hline Yes & 19 & 86.4 & 18 & 72.0 \\
\hline No & 3 & 13.6 & 7 & 28.0 \\
\hline \multicolumn{5}{|l|}{ Line of therapy } \\
\hline 0 & 0 & 0 & 1 & 4.0 \\
\hline 1 & 8 & 36.4 & 9 & 36.0 \\
\hline 2 & 5 & 22.7 & 7 & 28.0 \\
\hline$\geq 3$ & 9 & 40.9 & 8 & 32.0 \\
\hline \multicolumn{5}{|l|}{ Chemotherapy } \\
\hline Yes & 21 & 95.5 & 24 & 96.0 \\
\hline No & 1 & 4.5 & 1 & 4.0 \\
\hline \multicolumn{5}{|l|}{ Endocrine therapy } \\
\hline Yes & 16 & 72.7 & 18 & 72.0 \\
\hline No & 6 & 27.3 & 7 & 28.0 \\
\hline
\end{tabular}

Histology and receptor status refer to the primary tumor of the patient

NST invasive carcinoma of no special type, ILC invasive lobular carcinoma, $H R$ hormone receptor, $H E R 2$ human epidermal growth factor receptor 2, TNBC triple-negative breast cancer, PFS progression-free survival 


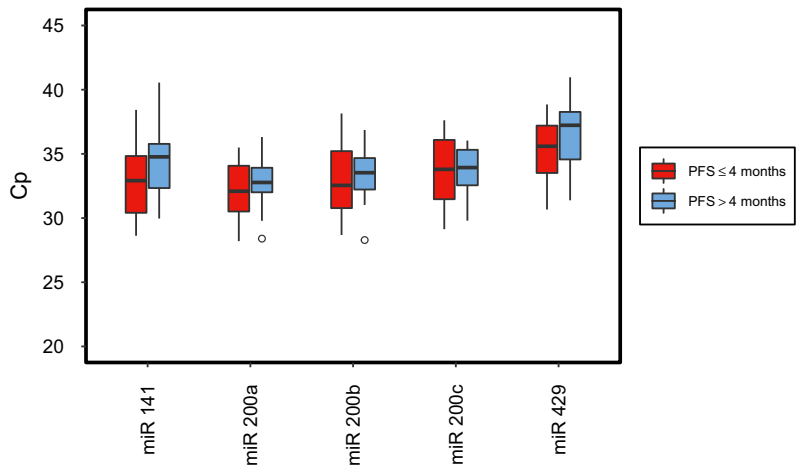

Fig. 1 Box plots showing miRNA levels of patients with $\mathrm{PFS} \leq 4$ months compared to patients with PFS $>4$ months before starting a new line of systemic therapy

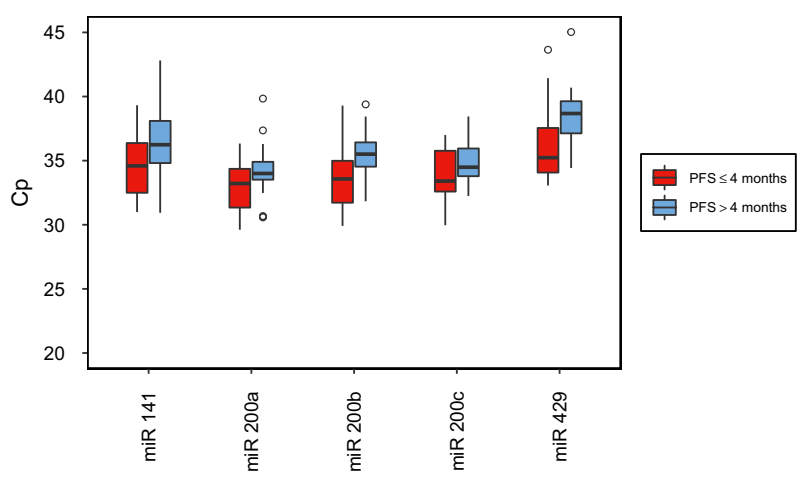

Fig. 2 Box plots showing miRNA levels of patients with $\mathrm{PFS} \leq 4$ months compared to patients with PFS $>4$ months after one cycle of a new line of systemic therapy

Table 2 Results of Student's $t$ test comparing miRNA expression in patients with early (PFS $\leq 4$ months, $n=22$ ) and late progression of disease (PFS $>4$ months, $n=25$ )

\begin{tabular}{llll}
\hline miRNA & & Baseline & $\begin{array}{l}\text { After } \\
\text { one cycle } \\
\text { of systemic } \\
\text { therapy } \\
p\end{array}$ \\
\hline miR-200a & $N$ & $p$ & 0.039 \\
miR-200b & 47 & 0.246 & 0.003 \\
miR-200c & 47 & 0.438 & 0.076 \\
miR-141 & 46 & 0.860 & 0.017 \\
miR-429 & 43 & 0.131 & 0.010 \\
\hline
\end{tabular}

$N$ : sample size, $p$ : statistical $p$ value

are of utmost importance to these patients' risk and thus, further clinical management.

Deductively speaking, this study not only provided evidence for the prognostic ability of the miR-200 family, but could further show that these miRNAs are of predictive
Table 3 Results of univariate logistic regression analysis of miRNA expression after systemic therapy in patients with PFS $\leq 4$ months as a predictor for early disease progression

\begin{tabular}{lll}
\hline miRNA & Estimate & $p$ \\
\hline miR-200a & 0.337 & 0.048 \\
miR-200b & 0.493 & 0.008 \\
miR-200c & 0.290 & 0.076 \\
miR-141 & 0.294 & 0.026 \\
miR-429 & 0.348 & 0.016 \\
\hline Estimate: & logistic & regression \\
coefficient, $p$ : statistical $p$ value
\end{tabular}

value for disease progression during systemic therapy and, thus, open up opportunities for clinical use as biomarkers in breast cancer management.

This analysis did not show any significant differences in miRNA expression levels among patients with early (PFS $\leq 4$ months) and late (PFS $>4$ months) PFS before starting a new line of systemic therapy. As patients were affected by metastatic progression at the time of study enrollment, which has been shown to be related to heightened miR-200s levels, it makes sense that miRNA expression patterns do not differ significantly between study groups $[9,11,18,19]$. In addition, differences in miRNA expression levels were observed in patients with local and bone metastases. It is crucial to note that the discordances were already observed at baseline and also seen after one cycle of therapy. A confounding capacity of said variables on the predictive value of miRNA on an early progression after therapy could not be confirmed by subsequent regression analysis. Remarkably, however, this study proved that after therapeutic intervention, the expression of 4 (miR-200a, miR-200b, miR-141, and miR-429) out of 5 members of the miR-200 family was significantly different in patients who suffered progression of disease within 4 months or less than in those who did not. In addition, these 4 miRNAs were found to be significant predictors of early relapse after therapeutic intervention. In line with these findings, other research showed a correlation of miR141, miR-200a, and miR-429 with Stage IV breast cancer and, even more interesting, suggested that high miR-141 serum expression is associated with shorter disease-free survival in metastatic cancer of the brain as well as a predictive value for decreased PFS [10, 20]. Furthermore, circulating miR-200c was recently shown to distinguish relapsed from non-relapsed patients with early breast cancer and, combined with lymph node infiltration, estrogen receptor status, and tumor grade, even to predict occurrence of late relapse [12]. Supporting the miR-200 cluster as clinically valuable biomarkers, another study reported elevated serum levels of miR-200a as predictive of resistance to chemotherapy in metastatic breast cancer patients, regardless of treatment regimen [13]. Moreover, Madhavan et al. reported that miR-200a, miR-200b, and miR-200c 
Fig. 3 Kaplan-Meier curves of miRNA prognostic groups and progression-free survival after one cycle of systemic therapy. Sample dichotomized as lower quartile (miRNA high levels) and upper rest (miRNA low levels) based on their $C p$ values
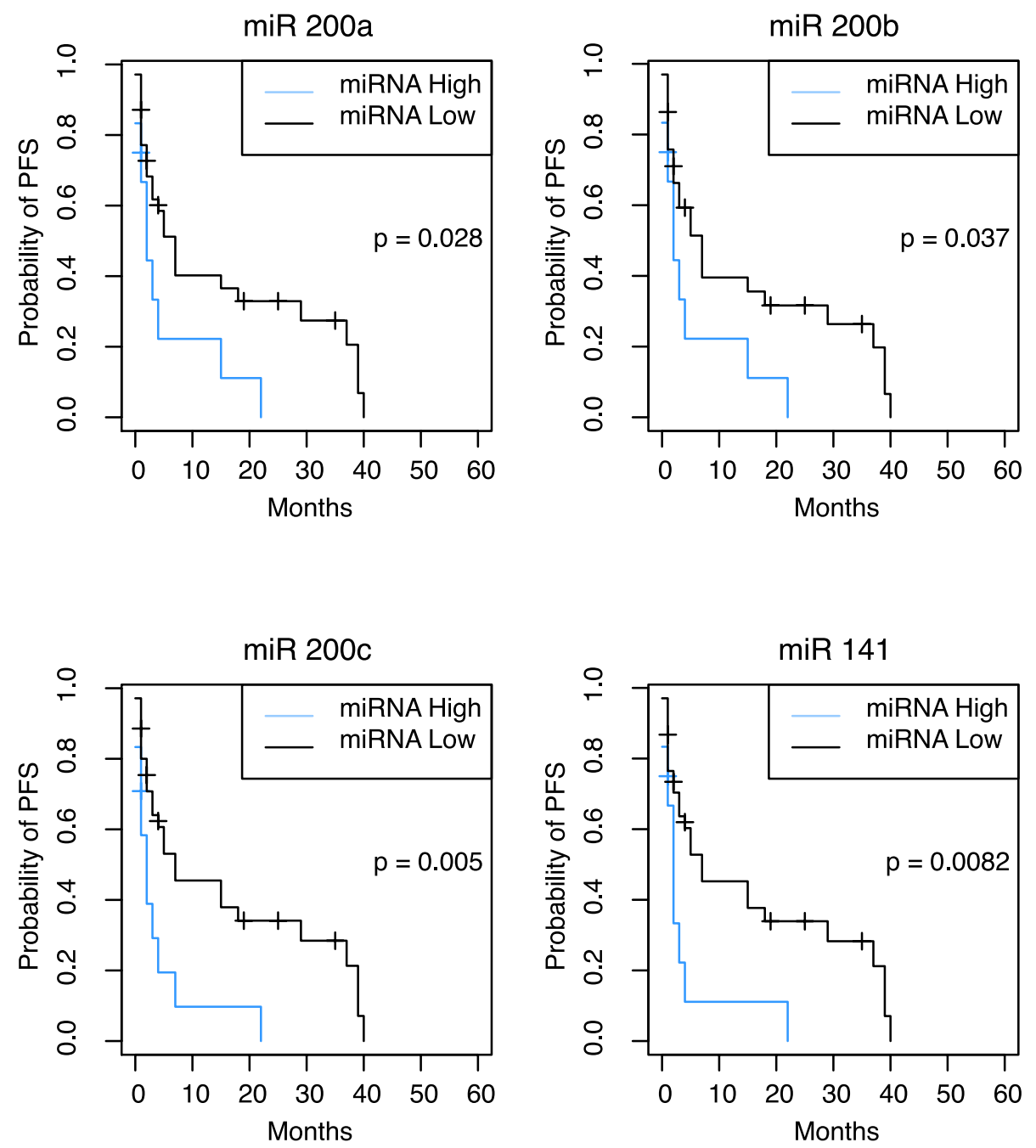

expression was predictive of metastatic onset, as upregulated plasma levels were measured 2 years before clinical diagnosis of breast cancer metastasis [21].

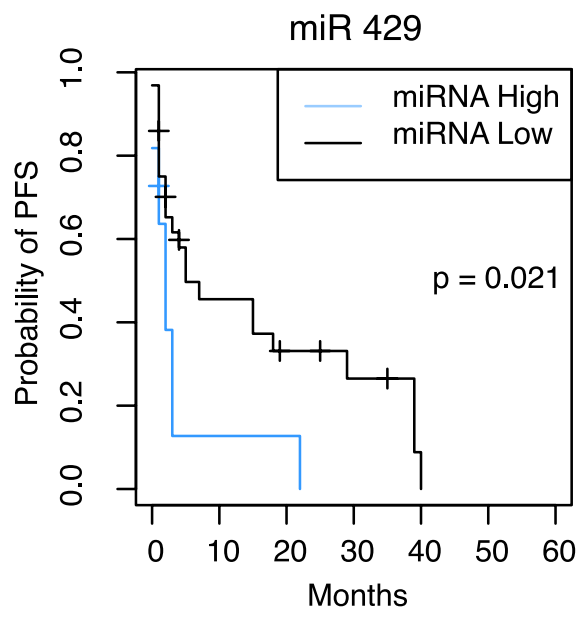

Since prognostic evaluations are of utmost importance for the further clinical management of these patients in terms of individualized treatment plans and follow-up for high-risk patients, our findings are especially intriguing. 
Fig. 4 Kaplan-Meier curves of miRNA prognostic groups and overall survival after one cycle of systemic therapy. Sample dichotomized as lower quartile (miRNA high levels) and upper rest (miRNA low levels) based on their $C p$ values
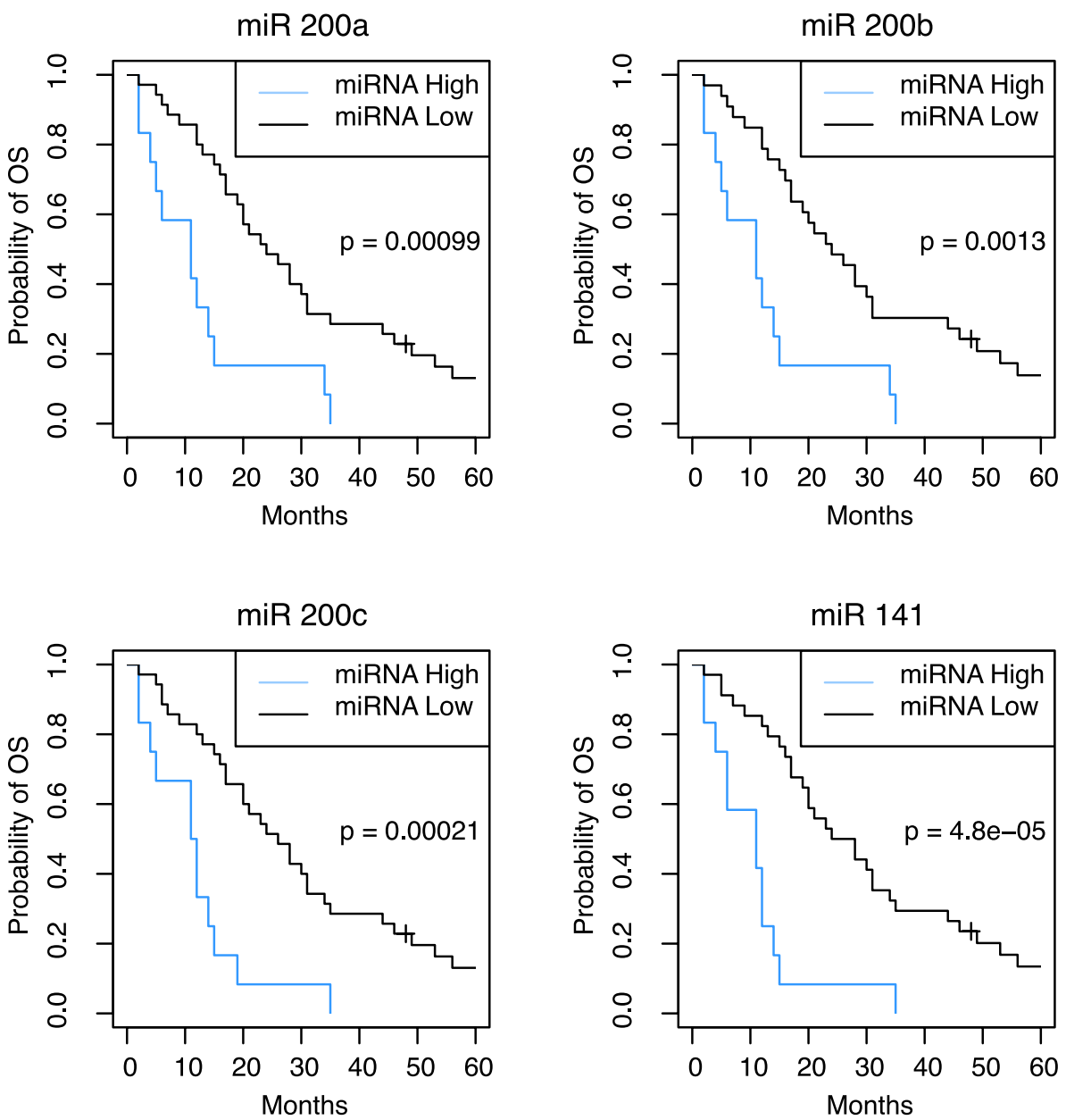

$\operatorname{miR} 429$

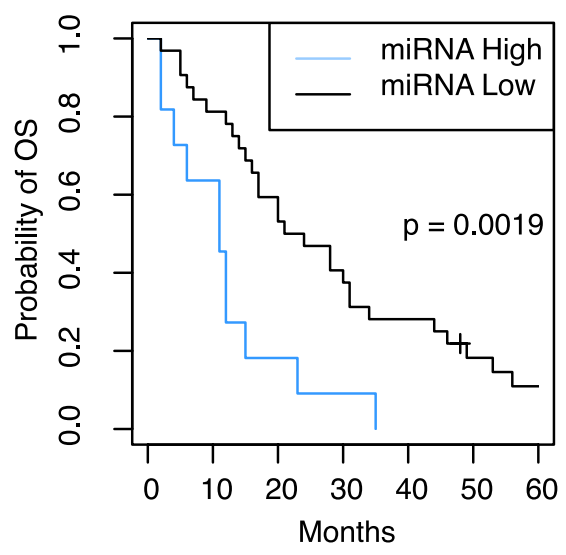

Although miR-200c did not quite reach the level of significance in terms of alpha 5\%, it is important not to neglect its involvement in the pathomechanisms of breast cancer, especially with regard to its genetic location. In humans, the miR-200 family is located at two sites of the genome: miR200a, miR-200b, and miR-141 form a cluster on chromosome 1 , and miR-200c and miR-141 build a cluster on chromosome 12 , respectively. Acknowledging, that miR-141 and miR-200c share the same genetic heritage and exert collective roles in the metastatic process, their respective, similar $p$ values are reasonable $\left(p_{\text {miR-141 }}=0.026, p_{\text {miR-200c }}=0.076\right)[17,22]$. In favor of this hypothesis, Dyxhoorn et al. reported that the miR141-200c cluster facilitates post extravasation events in lung cancer metastasis [6]. In addition, another study reported an association of this genetic location with biliary tract cancer [23]. When discussing and interpreting results based solely on 
Table 4 Results of univariate Cox-regression comparing OS and PFS distribution among miRNA prognostic groups after one cycle of systemic therapy

\begin{tabular}{|c|c|c|c|c|}
\hline \multirow[t]{2}{*}{ miRNA } & \multicolumn{2}{|l|}{$O S$} & \multicolumn{2}{|l|}{$P F S$} \\
\hline & HR (95\% CI) & $p$ & HR (95\% CI) & $p$ \\
\hline miR-200a & $3.12(1.54-6.31)$ & 0.001 & $2.25(1.05-4.82)$ & 0.037 \\
\hline miR-200b & $3.05(1.49-6.22)$ & 0.002 & $2.16(1.00-4.66)$ & 0.049 \\
\hline miR-200c & $3.70(1.79-7.62)$ & $<0.001$ & $2.81(1.33-5.93)$ & 0.007 \\
\hline miR-141 & $4.27(2.04-8.91)$ & $<0.001$ & $2.74(1.25-6.01)$ & 0.012 \\
\hline miR-429 & $3.09(1.47-6.47)$ & 0.003 & $2.50(1.11-5.65)$ & 0.028 \\
\hline
\end{tabular}

$O S$ overall survival, $P F S$ progression-free survival, $H R$ hazard ratio, $C I$ confidence interval, $p$ statistical $p$ value decision-making processes, thereby improving overall patient outcome and quality of life.

\section{Limitations}

Limiting factors of this study are the small sample size, which may have influenced the results and must be considered when interpreting the results. Unfortunately, due to the restricted number of cases and partly missing histological data, a valid statement about the association and expression of miR-200 expression in different tumor subtypes of this study collective is not possible. Regarding miRNA extraction, there is a lack of a universally accepted normalization method [29]. Therefore, $C p$ values of miRNA expression measured in plasma are subject to the limits of the quality of the respective normalization strategy. Finally, it should also be noted that $C p$ values above 35 may indicate inefficient miRNA extraction and should, therefore, be interpreted carefully.

$p$ values defined by dichotomous cutoff values, it is important not to overlook information that might possibly strengthen previous findings, such as the dual role of miR-141 and miR-200c in regulating metastasis [24]. It must also be borne in mind that the small sample size may have had an impact on the results. Taking these considerations into account, this study suggests that miR-200c and its predictive relation to PFS should not be disregarded.

Due to their ability to identify high-risk patients, miR200a, miR-200b, miR-141, and miR-429 are promising markers in the clinical setting for determining which patients in fact require and benefit from further, more intensive adjuvant therapeutic interventions as opposed to those who would be treated unnecessarily.

Given the still sparse data on the clinical use of miR-200s in metastatic breast cancer, this study further aims to improve the basic understanding of the dynamics of their expression patterns under therapy to contribute knowledge for a potential clinical application. Exemplarily, ongoing research showed that elevated miR-200 family levels correlate with decreased expression of the immune checkpoint protein PD-L1, thereby hypothesizing that measuring miR-200 levels might help identify patients with PD-L1 expression who would fit a respective therapy [25]. In addition, by interfering with the immune checkpoint protein expression, the miR-200 family might not only serve as a predictive marker but further be a potential therapeutic target and/or agent. [25-28]

Concluding, the presented results are of utmost clinical importance and need to be validated by further comprehensive studies, because as a predictive marker capable of identifying patients at high risk of disease progression, the miR-200 family has the potential to profoundly impact and improve clinical

\section{Conclusions}

Circulating miRNAs are differentially expressed in plasma of patients with late and/or early relapse. Four out of 5 members of the miR-200 family circulating in plasma predicted progression-free survival during systemic therapy. The miR-200 family is a valuable prognostic marker for overall and progression-free survival.

Supplementary Information The online version contains supplementary material available at https://doi.org/10.1007/s00404-022-06442-2.

Acknowledgements The authors gratefully acknowledge all patients whose data were used in this study. We also thank the medical and nursing staff at the National Center for Tumor Diseases (NCT; Heidelberg, Germany) for excellent management and care of the patients. This study was supported by the "Stiftung Landesbank Baden-Württemberg" and the "Stiftung für Krebs- und Scharlachforschung", made available to T.M. Deutsch. We acknowledge financial support by the Baden-Württemberg Ministry of Science, Research and the Arts and by Ruprecht-Karls-University Heidelberg.

Author contributions CF: project/protocol development, data collection, data management, data analysis, statistical analysis, manuscript writing/editing. TMD: project/protocol development, data collection, data management, data analysis, manuscript writing/editing. MF: data analysis, statistical analysis, manuscript editing. NR: data collection, data management, manuscript editing. FR: data collection, data management, manuscript editing. ADH: data analysis, manuscript editing. SYB: manuscript editing. CD: manuscript editing. CF: data collection, data management, manuscript editing. LM: data collection, data management, manuscript editing. BB: project/protocol development, methology development, manuscript editing. AS: project/protocol development, data collection, data management. AT: methology 
development, data analysis, manuscript editing. MW: project/protocol development, data collection, data management, data analysis, manuscript writing/editing.

Funding Open Access funding enabled and organized by Projekt DEAL. This study was supported by the "Stiftung Landesbank BadenWürttemberg" and the "Stiftung für Krebs- und Scharlachforschung", made available to T.M.D.. We acknowledge financial support by the Baden-Württemberg Ministry of Science, Research and the Arts and by Ruprecht-Karls-University Heidelberg.

Availability of data and materials The data sets generated during and/or analysed during the current study are available from the corresponding author on reasonable request.

Code availability Not applicable.

\section{Declarations}

Conflict of interest The authors declare no conflict of interest. The funders had no role in the design of the study; in the collection, analyses, or interpretation of data; in the writing of the manuscript, or in the decision to publish the results.

Ethical approval This study was performed in line with the principles of the Declaration of Helsinki 1964 and its later amendments. Approval was obtained by the Ethics Committee of the Medical Faculty Heidelberg of the Heidelberg University, approval No. S-295/2009.

Consent to participate Written informed consent was obtained from all individual participants included in the study.

Consent for publication All participants included in the study provided informed consent for publication of their data.

Open Access This article is licensed under a Creative Commons Attribution 4.0 International License, which permits use, sharing, adaptation, distribution and reproduction in any medium or format, as long as you give appropriate credit to the original author(s) and the source, provide a link to the Creative Commons licence, and indicate if changes were made. The images or other third party material in this article are included in the article's Creative Commons licence, unless indicated otherwise in a credit line to the material. If material is not included in the article's Creative Commons licence and your intended use is not permitted by statutory regulation or exceeds the permitted use, you will need to obtain permission directly from the copyright holder. To view a copy of this licence, visit http://creativecommons.org/licenses/by/4.0/.

\section{References}

1. Bray F, Ferlay J, Soerjomataram I et al (2018) Global cancer statistics 2018: GLOBOCAN estimates of incidence and mortality worldwide for 36 cancers in 185 countries. CA Cancer J Clin 68:394-424. https://doi.org/10.3322/caac. 21492

2. Krebs in Deutschland für 2013/2014. 11. Ausgabe. Robert KochInstitut (Hrsg) und die Gesellschaft der epidemiologischen Krebsregister in Deutschland e.V. (Hrsg). Berlin, 2017. https://www. krebsdaten.de/Krebs/DE/Content/Publikationen/Krebs_in_Deuts chland/kid_2017/krebs_in_deutschland_2017.pdf?
3. de Rubis G, Rajeev Krishnan S, Bebawy M (2019) Liquid biopsies in cancer diagnosis, monitoring, and prognosis. Trends Pharmacol Sci 40:172-186. https://doi.org/10.1016/j.tips.2019.01.006

4. Bartel DP (2004) MicroRNAs: genomics, biogenesis, mechanism, and function. Cell 116:281-297

5. Senfter D, Madlener S, Krupitza G et al (2016) The microRNA-200 family: still much to discover. Biomol Concepts 7:311319. https://doi.org/10.1515/bmc-2016-0020

6. Dykxhoorn DM, Wu Y, Xie H et al (2009) miR-200 enhances mouse breast cancer cell colonization to form distant metastases. PLoS ONE 4:e7181. https://doi.org/10.1371/journal.pone.00071 81

7. Korpal M, Ell BJ, Buffa FM et al (2011) Direct targeting of Sec23a by miR-200s influences cancer cell secretome and promotes metastatic colonization. Nat Med 17:1101-1108. https:// doi.org/10.1038/nm.2401

8. Markou A, Zavridou M, Sourvinou I et al (2016) Direct comparison of metastasis-related miRNAs expression levels in circulating tumor cells, corresponding plasma, and primary tumors of breast cancer patients. Clin Chem 62:1002-1011

9. Madhavan D, Zucknick M, Wallwiener M et al (2012) Circulating miRNAs as surrogate markers for circulating tumor cells and prognostic markers in metastatic breast cancer. Clin Cancer Res 18:5972-5982. https://doi.org/10.1158/1078-0432.CCR-12-1407

10. Debeb BG, Lacerda L, Anfossi S et al (2016) miR-141-mediated regulation of brain metastasis from breast cancer. J Natl Cancer Inst. https://doi.org/10.1093/jnci/djw026

11. Papadaki C, Stoupis G, Tsalikis L et al (2019) Circulating miRNAs as a marker of metastatic disease and prognostic factor in metastatic breast cancer. Oncotarget 10:966-981. https://doi.org/ 10.18632/oncotarget.26629

12. Papadaki C, Stratigos M, Markakis G et al (2018) Circulating microRNAs in the early prediction of disease recurrence in primary breast cancer. Breast Cancer Res 20:72. https://doi.org/10. 1186/s13058-018-1001-3

13. Shao B, Wang X, Zhang L et al (2019) Plasma microRNAs predict chemoresistance in patients with metastatic breast cancer. Technol Cancer Res Treat 18:1533033819828709. https://doi.org/10.1177/ 1533033819828709

14. Eisenhauer EA, Therasse P, Bogaerts J et al (2009) New response evaluation criteria in solid tumours: revised RECIST guideline (version 1.1). Eur J Cancer 45:228-247. https://doi.org/10.1016/j. ejca.2008.10.026

15. Turchinovich A, Weiz L, Langheinz A et al (2011) Characterization of extracellular circulating microRNA. Nucleic Acids Res 39:7223-7233. https://doi.org/10.1093/nar/gkr254

16. R Foundation for Statistical Computing R (2022) A language and environment for statistical computing. http://www.r-project.org/ index.html

17. Humphries B, Yang C (2015) The microRNA-200 family: small molecules with novel roles in cancer development, progression and therapy. Oncotarget 6:6472-6498. https://doi.org/10.18632/ oncotarget. 3052

18. Zhang G, Zhang W, Li B et al (2017) MicroRNA-200c and microRNA-141 are regulated by a FOXP3-KAT2B axis and associated with tumor metastasis in breast cancer. Breast Cancer Res 19:73. https://doi.org/10.1186/s13058-017-0858-X

19. Yu S-J, Hu J-Y, Kuang X-Y et al (2013) MicroRNA-200a promotes anoikis resistance and metastasis by targeting YAP1 in human breast cancer. Clin Cancer Res 19:1389-1399. https://doi.org/10. 1158/1078-0432.CCR-12-1959

20. Fontana A, Barbano R, Dama E et al (2021) Combined analysis of miR-200 family and its significance for breast cancer. Sci Rep 11:2980. https://doi.org/10.1038/s41598-021-82286-1

21. Madhavan D, Peng C, Wallwiener M et al (2016) Circulating miRNAs with prognostic value in metastatic breast cancer and for 
early detection of metastasis. Carcinogenesis 37:461-470. https:// doi.org/10.1093/carcin/bgw008

22. Gregory PA, Bert AG, Paterson EL et al (2008) The miR-200 family and miR-205 regulate epithelial to mesenchymal transition by targeting ZEB1 and SIP1. Nat Cell Biol 10:593-601. https://doi. org/10.1038/ncb1722

23. Meng F, Henson R, Lang M et al (2006) Involvement of human micro-RNA in growth and response to chemotherapy in human cholangiocarcinoma cell lines. Gastroenterology 130:2113-2129. https://doi.org/10.1053/j.gastro.2006.02.057

24. Amrhein V, Korner-Nievergelt F, Roth T (2017) The earth is flat (p 0.05): significance thresholds and the crisis of unreplicable research. PeerJ 5:e3544. https://doi.org/10.7717/peerj.3544

25. Noman MZ, Janji B, Abdou A et al (2017) The immune checkpoint ligand PD-L1 is upregulated in EMT-activated human breast cancer cells by a mechanism involving ZEB-1 and miR-200. Oncoimmunology 6:e1263412. https://doi.org/10.1080/21624 02X.2016.1263412

26. Smolle MA, Prinz F, Calin GA et al (2019) Current concepts of non-coding RNA regulation of immune checkpoints in cancer.
Mol Aspects Med 70:117-126. https://doi.org/10.1016/j.mam. 2019.09.007

27. Smolle MA, Calin HN, Pichler M et al (2017) Noncoding RNAs and immune checkpoints-clinical implications as cancer therapeutics. FEBS J 284:1952-1966. https://doi.org/10.1111/febs.14030

28. Shukuya T, Ghai V, Amann JM et al (2020) Circulating MicroRNAs and extracellular vesicle-containing MicroRNAs as response biomarkers of anti-programmed cell death protein 1 or programmed death-ligand 1 therapy in NSCLC. J Thorac Oncol 15:1773-1781. https://doi.org/10.1016/j.jtho.2020.05.022

29. Schwarzenbach H, da Silva AM, Calin G et al (2015) Data normalization strategies for MicroRNA quantification. Clin Chem 61:1333-1342. https://doi.org/10.1373/clinchem.2015.239459

Publisher's Note Springer Nature remains neutral with regard to jurisdictional claims in published maps and institutional affiliations. 